\title{
Sequence to Sequence Learning for Query Expansion
}

\author{
Salah Zaiem \\ Ecole Polytechnique \\ Palaiseau, France \\ mohamed-salah.zaiem@polytechnique.edu
}

\author{
Fatiha Sadat \\ Universite du Quebec a Montreal \\ 201 Avenue du Président Kennedy, Montreal \\ sadat.fatiha@uqam.ca
}

\begin{abstract}
As fas as we are aware, using Sequence to Sequence algorithms for query expansion has not been explored yet in Information Retrieval literature. We tried to fill this gap in the literature with a custom Query Expansion system trained and tested on open datasets. One specificity of our engine compared to classic ones is that it does not need the documents to expand the introduced query. We test our expansions on two different tasks : Information Retrieval and Answer preselection. Our method yielded a slight improvement in performance in both two tasks. Our main contributions are :

- Starting from open datasets, we built a Query Expansion training set using sentence-embeddings-based Keyword Extraction.

- We assess the ability of the Sequence to Sequence neural networks to capture expanding relations in the words embeddings' space.

We afterwards started a quantitative and qualitative analysis of the weights learned by our network. In the second part, I will discuss what is learned by a Recurrent Neural Network compared to what we know about human language learning.
\end{abstract}

\section{Related Work}

Relevance feedback has been a popular choice for query expansion, starting with the Rocchio Algorithm (Salton 1971) in SMART Information Retrieval System. Using a set of relevant and non relevant documents, the original query vector is modified.

Recently, the introduction of word embeddings (Mikolov et al. 2013) allowed new possibilities for Query Expansion. The distributed representations of the words in a query made it possible to produce expansions without extracting them from the documents. Using the centroid of the words introduced and cosine-similar tokens, Kuzi and al (2016) proposed a document-independent expansion method.

\section{Sequence to sequence architectures}

Sequence to Sequence is a neural architecture very popular in machine translation since it has achieved state of the art

Copyright (c) 2019, Association for the Advancement of Artificial Intelligence (www.aaai.org). All rights reserved. results. Proposed by Sutskever and al (2014), it consists in a two-component model using recurrent neural networks to link variable-length input sequences to variable-length output sequences. The introduced sequence gets encoded by the first component into a vectorial representation. Therefore, the decoder transforms that vector into the target sequence. At each step the next token maximizes :

$$
p\left(y_{i} \mid y_{1}, \ldots, y_{i-1}, x\right)=g\left(y_{i-1}, s_{i}, c\right)
$$

where $s_{i}$ is the $\mathrm{i}$-th hidden state of the encoder, $\mathrm{c}$ the final vector output by the encoder representing the entire input sentence and $y_{i}$ the $\mathrm{i}$-th generated token. $\mathrm{g}$ is the function learned by the decoder.

\section{Our Approach \\ Building the training set}

Datasets We used MultiNLI (Williams, Nangia, and Bowman 2018) and SNLI (Bowman et al. 2015). For both corpora, we naturally eliminate pairs classified as contradiction as they shall not provide relevant expansions.

We also selected the duplicate pairs from the Quora question pairs dataset and trained our expansion model using the words that do not appear in the first formulation.

Finally, MSCOCO (Lin et al. 2014) dataset consists in human annotated captions of over $120 \mathrm{~K}$ images. Since they are describing the same image. We can assume the words appearing in one description and not in the other are an eventual expansion for the first annotation.

Keywords extraction We will use sentence embeddings to find out which words contribute most to the final vector. This computation is based on the hidden states of the encoder. The last layer in the Infersent (Conneau et al. 2017) model is a Maxpooling one. The words chosen the most times will be our selected keywords.

\section{The training}

Preprocessing We extract the keywords from the target sequence, and then we remove the ones that appear in the source sequence. To get targets with similar lengths, we remove the pairs with a target having less than 3 tokens and limit them to 6 . We finally get $520 \mathrm{k}$ pairs of sentenceexpansion. 
Training Model We initiated the encoder and decoder weights with pre-trained word embeddings. We chose Glove $840 \mathrm{~B}$ with 300 dimensioned vectors. To choose the hyper parameters, we relied mainly on the best practices given by Britz and al. (2017). We used a Bidirectional LSTM encoder with two layers of 500 hidden units. The decoder is a 2-layer LSTM with 500 hidden units.

We used mini batches of 32 examples, and applied a 0.35 dropout probability in the LSTM stacks. We used Stochastic Gradient Descent as our optimizer and started with a learning rate of 0.001 . The learning rate goes down with a decay of 0.5 after every epoch. There were 25 epochs of training for a total training time of 85 hours. The loss function is a Softmax cross entropy loss.

\section{Evaluation}

We tested our query expansion model on two different tasks: Information Retrieval and Answer preselection.

\section{Information Retrieval}

For this task, we will use the TREC Robust 2004 Dataset . It consists in a set of 250 queries and 528,155 documents. For the search component, we will use Apache Lucene search.

We start with the queries, and we expand them using our QE system and then we check the quality of the results provided by Lucene Search using StandardAnalyzer and two different weighting schemes : TF-IDF and BM25.

\begin{tabular}{|l|c|}
\hline Method & MAP \\
\hline TF-IDF without QE & 0.2517 \\
TF-IDF with QE & 0.2581 \\
BM25 without QE & 0.2709 \\
BM25 with QE & 0.2783 \\
\hline
\end{tabular}

Table 1: Information Retrieval results

\section{Answer Preselection}

We used the WikiQA Dataset (Yang, Yih, and Meek 2015). For each question, we start a search on the set of answers with a similarity computation. Accuracy is the proportion of relevant answers within the ten preselected hypothesis. Coverage is defined as the proportion of queries that had at least one appropriate answer among the ten hypotheses.

\begin{tabular}{|l|r|l|}
\hline Method & Accuracy & Coverage \\
\hline TF-IDF without QE & 0.2871 & 0.7840 \\
TF-IDF with QE & 0.2889 & 0.7901 \\
\hline
\end{tabular}

Table 2: Answer Preselection results

\section{Qualitative analysis and future tracks}

Our Query Expansion engine does improve the results in the different tasks we tested it in, but the progress is far from being impressive, and is logically not statistically significant.
Here is a list of the issues limiting the reliability of our QE system and a few future tracks for improvement :

- The QE system fails to capture the semantic mechanisms behind Query Expansion, and therefore could not expand queries of unseen topics. This may not be that surprising as the task seems very complicated and nothing proofs that the actual embedding space ensures and holds this type of semantic relationships. The expansions are therefore learned through the examples, and the models fails to enrich queries on topics it did not witness before ( $45 \%$ of the queries are not expanded since no new word is added).

- This makes us think that although it may not be efficient for open topics, training this model on local entailments would yield great expansion results.

- We will explore the possibility of including the search in the training process. The progress on search would be a loss function updating the weights of the encoder-decoder network. After the first training, a second one ,based on the reward for the search, would refine the parameters of our network and make it more search-oriented. With more training data, we can expect an improvement in terms of precision (MAP) and accuracy.

\section{References}

Bowman, S. R.; Angeli, G.; Potts, C.; and Manning, C. D. 2015. A large annotated corpus for learning natural language inference. In EMNLP 2015, 632-642.

Britz, D.; Goldie, A.; Luong, M.-T.; and Le, Q. 2017. Massive exploration of neural machine translation architectures. In EMNLP 2017, 1442-1451.

Conneau, A.; Kiela, D.; Schwenk, H.; Barrault, L.; and Bordes, A. 2017. Supervised learning of universal sentence representations from natural language inference data. In EMNLP 2017, 670-680.

Kuzi, S.; Shtok, A.; and Kurland, O. 2016. Query expansion using word embeddings. In CIKM'16, 1929-1932. New York, NY, USA: ACM.

Lin, T.; Maire, M.; Belongie, S. J.; Bourdev, L. D.; Girshick, R. B.; Hays, J.; Perona, P.; Ramanan, D.; Dollár, P.; and Zitnick, C. L. 2014. Microsoft COCO: common objects in context. CoRR abs/1405.0312.

Mikolov, T.; Sutskever, I.; Chen, K.; Corrado, G. S.; and Dean, J. 2013. Distributed representations of words and phrases and their compositionality.

Salton, G. 1971. The SMART Retrieval SystemExperiments in Automatic Document Processing.

Sutskever, I.; Vinyals, O.; and Le, Q. V. 2014. Sequence to sequence learning with neural networks. In NIPS'14.

Williams, A.; Nangia, N.; and Bowman, S. 2018. A broad-coverage challenge corpus for sentence understanding through inference. In NAACL-HLT 2018, 1112-1122.

Yang, Y.; Yih, W.-t.; and Meek, C. 2015. Wikiqa: A challenge dataset for open-domain question answering. In Proceedings of the 2015 EMNLP Conference, 2013-2018. 